\title{
Genes and pathways associated with the occurrence of malignancy in benign lymphoepithelial lesions
}

\author{
YAO MAWULIKPLIMI ADZAVON ${ }^{1 *}$, PENGXIANG ZHAO ${ }^{1 *}$, XIN ZHANG $^{1}$, MENGYU LIU $^{1}$, BAOBEI LV ${ }^{1}$, \\ LINQI YANG ${ }^{1}$, XUJUAN ZHANG $^{1}$, FEI XIE $^{1}$, MINGZI ZHANG ${ }^{2}$, JIANMIN MA $^{3}$ and XUEMEI MA ${ }^{1}$ \\ ${ }^{1}$ College of Life Science and Bio-Engineering, Beijing University of Technology, Beijing 100124; \\ ${ }^{2}$ Department of Plastic Surgery, Peking Union Medical College Hospital; ${ }^{3}$ Beijing Ophthalmology and Vision Science \\ Key Lab, Beijing Tongren Eye Center, Beijing Tongren Hospital, Capital Medical University, Beijing 100730, P.R. China
}

Received May 16, 2017; Accepted September 6, 2017

DOI: $10.3892 / \mathrm{mmr} .2017 .8149$

\begin{abstract}
There is increasing evidence concerning the occurrence of malignant lymphoma among people suffering from Mikulicz disease, also termed benign lymphoepithelial lesion (BLEL) and immunoglobulin G4-associated disease. However, the underlying molecular mechanism of the malignant transformation remains unclear. The present study aimed to investigate the gene expression profile between BLEL and malignant lymphoepithelial lesion (MLEL) conditions using tissue microarray analysis, to identify genes and pathways which may be associated with the risk of malignant transformation. Comparing gene expression profiles between BLEL tissues $(n=13)$ and MLEL $(n=14)$, a total of 1,002 differentially expressed genes (DEGs) were identified including 364 downregulated and 638 upregulated DEGs in BLEL. The downregulated DEGs in BLEL were frequently associated with immune-based functions, immune cell differentiation, proliferation and survival, and metabolic functions, whereas the upregulated DEGs were primarily associated with organ, gland and tissue developmental processes. The B cell receptor signaling pathway, the transcription factor p65 signaling pathway, low affinity immunoglobulin $\gamma \mathrm{Fc}$ region receptor II-mediated phagocytosis, the high affinity immunoglobulin $\varepsilon$ receptor subunit $\gamma$ signaling pathway and Epstein-Barr virus infection, and pathways in cancer, were the pathways associated with the downregulated DEGs. The upregulated DEGs were associated with three pathways,
\end{abstract}

Correspondence to: Dr Xuemei Ma or Dr Pengxiang Zhao, College of Life Science and Bio-Engineering, Beijing University of Technology, 100 Pingle Yuan, Beijing 100124, P.R. China

E-mail:xmma@bjut.edu.cn

E-mail: zpx@bjut.edu.cn

*Contributed equally

Key words: benign lymphoepithelial lesion, inflammation, malignant B lymphoma, malignant lymphoepithelial lesion, risk of malignancy including glutathione metabolism, salivary secretion and mineral absorption pathways. These results suggested that the identified signaling pathways and their associated genes may be crucial for understanding the molecular mechanisms underlying malignant transformation from BLEL, and they may be considered to be markers for predicting malignancy among the BLEL group.

\section{Introduction}

Benign lymphoepithelial lesion (BLEL), also termed Mikulicz disease, is a systemic inflammatory disease of unknown origin. It is characterized by bilateral and persistent enlargement of lacrimal and salivary glands caused by infiltration of immunoglobulin (Ig)G4-positive plasma cells in the gland tissues $(1,2)$. Initially assumed to be Sjögren's syndrome due to their histopathological similarities, Mikulicz disease has been established as a novel clinical entity. As a result of the elevated IgG4 concentration in serum in addition to the tumor-like swelling of involved organs and their variable degrees of fibrosis, Mikulicz disease has been included among the IgG4-associated diseases and recognized as immune-mediated conditions (1-3).

A number of cases and types of lymphoma have been reported in patients with BLEL however it is difficult to estimate the exact rate of lymphoma occurrence because of the low prevalence of malignant transformation. Nevertheless, a rate of $10.4 \%$ (11/106 patients) of malignancies was observed by Yamamoto et al (4) in 2012, 12\% (2/17) by Sato et al (5) in 2008 , and $14.3 \%$ (2/14) by Go et al (6) in 2012. Additionally, increasing numbers of studies highlighting evidence of malignant lymphoma among patients with Mikulicz disease and IgG4-associated disorders are accumulating. In 1971, Azzopardi et al (7) reported on five patients who exhibited signs of Mikulicz disease and malignant lymphoma at the same site; years later, three cases of possible malignant transformation of BLEL in human immunodeficiency virus-infected patients were reported (8); three other cases of malignant lymphoma, which arose in the parotid and the lacrimal glands of patients with BLEL, were reported by Sato et al (9) in 2002. More recently, marginal zone B cell lymphoma-mimicking IgG4-associated dacryoadenitis and sialoadenitis (10), or 
ocular adnexal marginal zone lymphomas arising from association with IgG4-associated disease, were reported (11). All these observations suggested that patients with BLEL, in addition to people suffering from IgG4-associated disease, exhibit a markedly increased risk for developing malignant lymphoma. Although, the risk of malignancy had been highlighted in BLEL and IgG4-associated disorders, the mechanisms underlying this malignant transformation remain unknown. The present study aimed to identify the molecular mechanisms, which may be involved in this malignant transformation by analyzing gene expression profiles between the two conditions.

\section{Materials and methods}

Sampling and microarray data processing. Following signed consent being obtained from all patients, tissue biopsies were obtained at Beijing Tongren Hospital between December 2011 and April 2012 from an adult population (36-78 years old, with a male:female ratio of 1:1.25) with the approval of the Local Ethics Committee of Beijing Tongren Hospital, Capital Medical University (Beijing, China). Biopsies of 14 fresh orbital tissues of malignant lymphoepithelial lesions (MLELs) composed of malignant $\mathrm{B}$ lymphoma patients (nine mucosa-associated lymphoid tissue B lymphomas, two large B lymphomas and three other B lymphomas) and 13 orbital tissues from patients with BLEL were subjected to Phalanx Human OneArray ${ }^{\circledR}$ Gene Expression Profiling (Phalanx Biotech Group, Hsinchu, Taiwan), according to the manufacturer's protocol. All the data were analyzed in duplicate. Following this, a robust multi-array average algorithm in the Affy package (Bioconductor; bioconductor.org/packages/release/bioc/html/affy.html) was used with $\mathrm{R}$ software ( $\mathrm{R}$ version 3.3.0; www.r-project.org) for background correction, quantile normalization, and for summarization of the expression measure for each probe set in each array.

Identification and clustering of differentially expressed genes (DEGs). A linear model for microarray data analysis provided by Bioconductor was fitted to the obtained gene expression matrix, which was used to identify the DEGs between BLEL and MLEL. The Benjamini-Hochberg procedure for multiple testing correction was used to control the false discovery rate (FDR) and significant DEGs were selected with $\mid \log 2 \mathrm{FCl}>1$ and $\mathrm{FDR}<0.01$. Clustering analysis and heatmap creation for the DEGs were performed using dChip software version $2011.01(12,13)$.

Protein-protein interaction (PPI) network construction for the DEGs. In living cells, molecular and biological processes are regulated by PPIs. PPIs are useful resources to identify novel pathways in order to gain a basic knowledge of diseases. Therefore, PPI were assessed for down- and upregulated DEGs with the online Search Tool for the Retrieval of Interacting Genes platform (version 10.0; https://string-db. org/). The highest confidence score $\geq 0.9$ was used to predict links between proteins involved in the network and visualization of the interaction network was performed using Cytoscape (Cytoscape version 3.4.0; http://www.cytoscape. org/).
Functional annotation and pathway enrichment. Gene ontology (GO) functional annotation for biological process and the Kyoto Encyclopedia of Genes and Genomes (KEGG) pathways enrichment for the DEGs between MLEL and BLEL were performed using version 6.8 of the Database for Annotation, Visualization and Integrated Discovery (DAVID) online platform; https://david.ncifcrf.gov/. The Benjamini-Hochberg procedure for multiple testing correction was used to control the FDR. Significantly enriched GO terms and KEGG pathways were selected with an FDR $<0.05$.

Statistical analysis. Quantitative data were analyzed using GraphPad Prism v.5 (GraphPad Software, Inc., La Jolla, CA, USA) and are plotted as the mean \pm standard error of the mean. For group comparisons, one-way analysis of variance with Bonferroni's multiple comparison test was used. $\mathrm{P}<0.05$ was considered to indicate a statistically significant difference.

\section{Results}

Differential gene expression and expression pattern between $B L E L$ and MLEL. Gene expression profiling between BLEL $(\mathrm{n}=13)$ and MLEL $(\mathrm{n}=14)$ biopsy tissues were performed for a total of 20,138 genes, and significant DEGs between the BLEL group and MLEL group were selected with $\mid \log 2 \mathrm{FCl}>1$ and FDR $<0.01$. A total of 1,002 genes $(4.98 \%)$ were identified to be differentially expressed. Compared with the MLEL samples, 364 genes were downregulated and 638 genes upregulated in the BLEL group.

The results of the DEG visualization and selection, and the hierarchical clustering of the DEGs between the BLEL and MLEL groups, are exhibited for each replicate in Fig. 1.

PPI network analysis of the DEGs. The analysis of the PPIs for up- and downregulated DEGs with a confidence score $\geq 0.9$ revealed that 62 proteins from the downregulated DEGs in the BLEL samples were engaged in the PPI networks, with a total of 91 interactions (Fig. 2). For the upregulated DEGs, 216 interactions were observed among the 156 proteins implicated in the PPI network (Fig. 3).

Functional annotation of DEGs in the PPI network. The downregulated DEGs in the BLEL group compared with the MLEL group were primarily increased in biological processes associated with immune signaling functions, including signal transduction, B cell activation and differentiation, response to stimulus and immune system process, while the upregulated DEGs were primarily associated with development-associated signaling processes, including cells and cellular components movement, response to stimulus, gland and tissues development, biological adhesion and cell migration (Tables I and II).

Pathway enrichment of the DEGs in the PPI networks. KEGG pathways enrichment for the DEGs in the PPI network was performed using the online functional annotation tool DAVID. With a significant cut-off fixed at FDR $<0.05$, six KEGG pathways were significantly enriched for the downregulated DEGs and three for the upregulated DEGs (Table III). The downregulated DEGs in BLEL were associated with the B cell receptor (BCR) signaling pathway, 

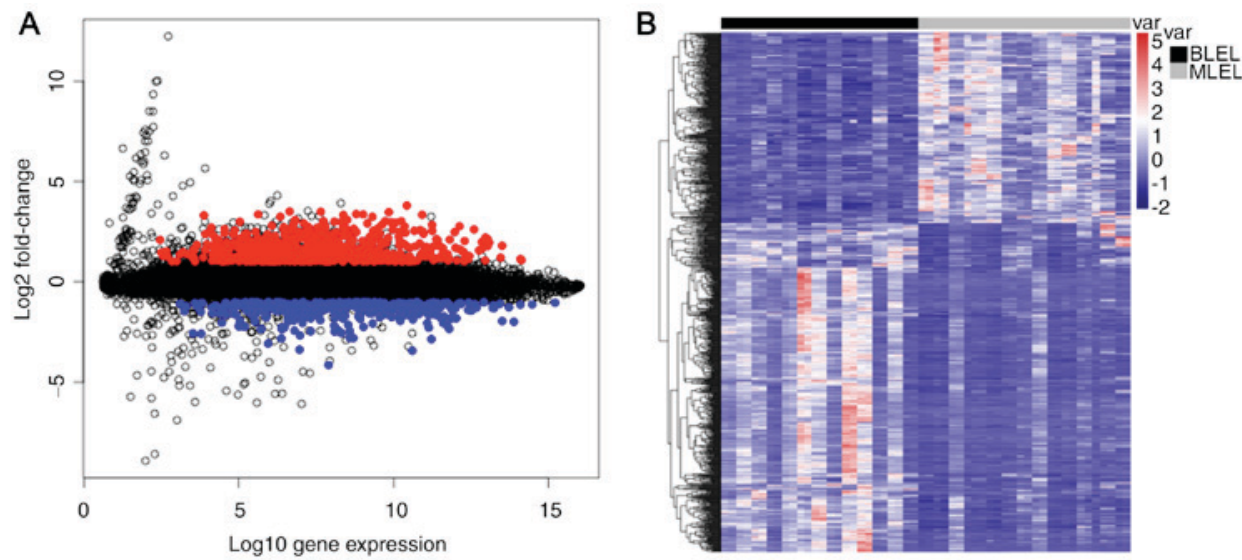

Figure 1. DEGs between the BLEL and MLEL groups. (A) MA-plot of DEGs, with red dots and blue dots representing the upregulated genes and downregulated genes, respectively. (B) Heatmap of DEGs. The x-axis represents the tissue samples from BLEL (black) and MLEL (grey), and y-axis represents the screened genes. MLEL, malignant lymphoepithelial lesion; BLEL; benign lymphoepithelial lesion; DEGs, differentially expressed genes.
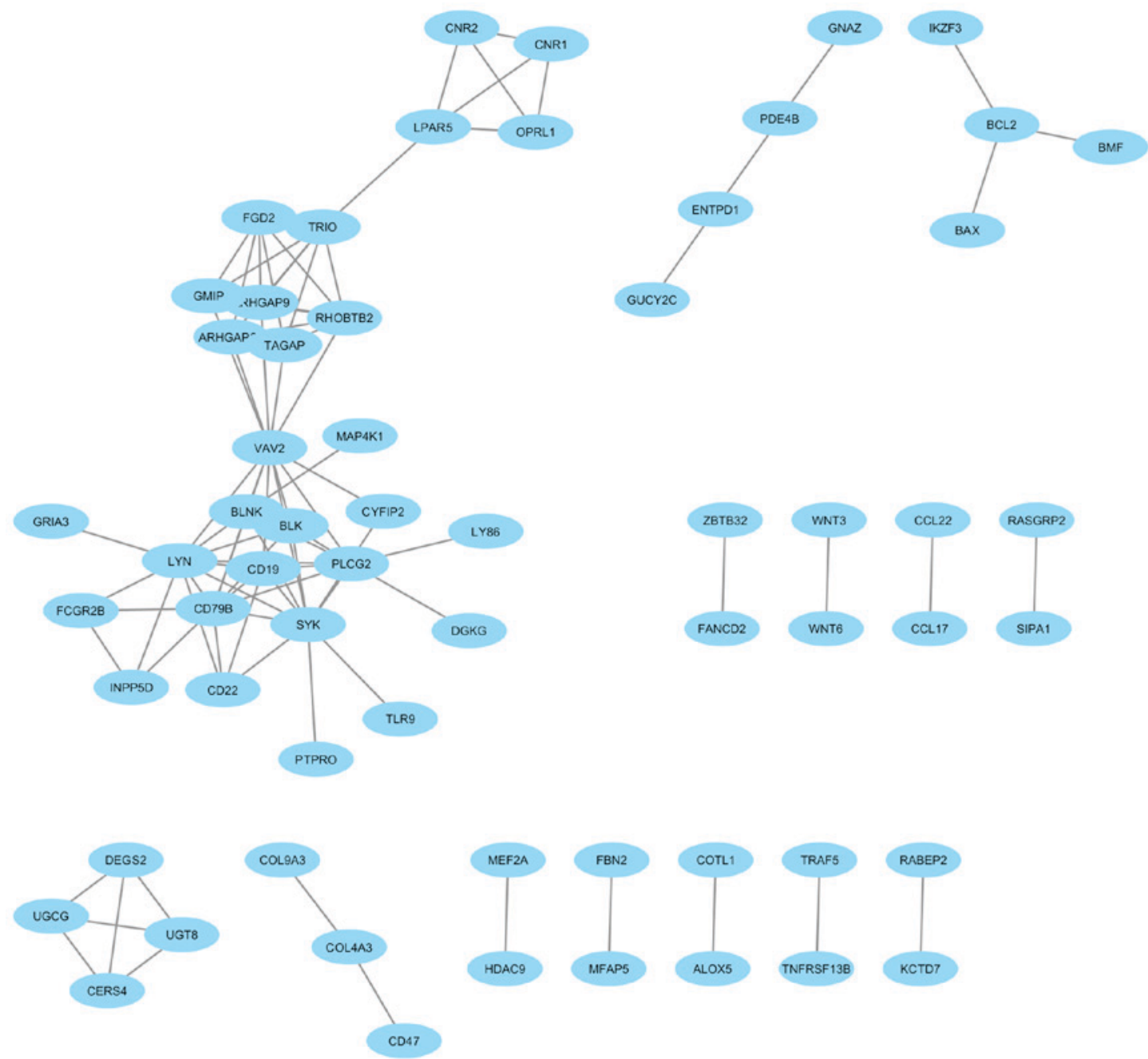

Figure 2. Protein-protein interaction network of downregulated DEGs. Nodes (in blue) represent DEGs and the lines (in grey) between nodes represent interactions. DEGs, differentially expressed genes.

transcription factor p65 signaling pathway, low affinity immunoglobulin $\gamma$ Fc region receptor II-mediated phagocytosis, high affinity immunoglobulin $\varepsilon$ receptor subunit $\gamma$ signaling pathway, Epstein-Barr virus infection pathway and pathways in cancer. Glutathione metabolism, salivary secretion and the mineral absorption pathway were the only notable pathways associated with the upregulated DEGs. A map of the DEGs with the KEGG pathways for the BCR signaling pathway and pathways in cancer are exhibited in Figs. 4 and 5, respectively. 


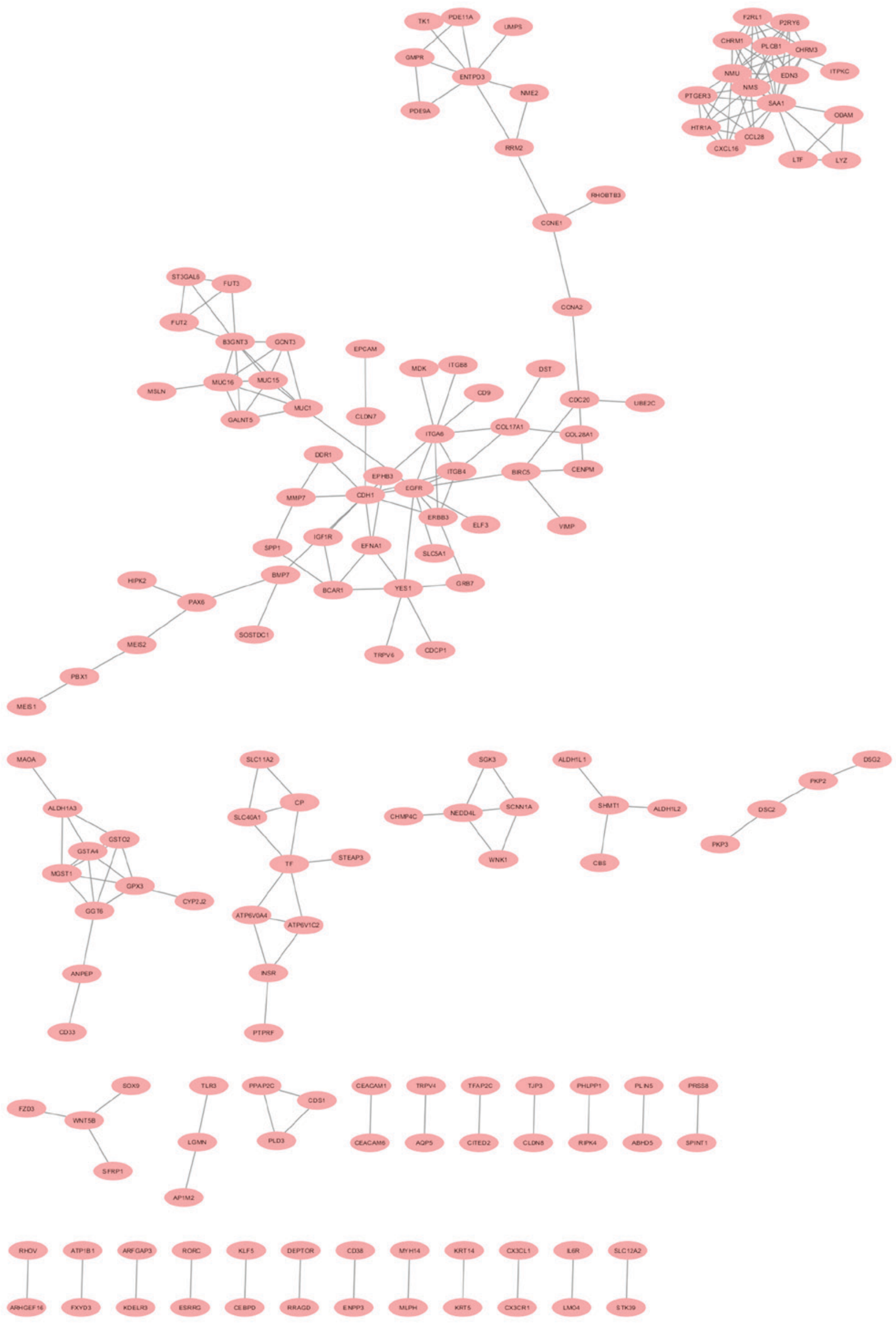

Figure 3. Protein-protein interaction network of upregulated DEGs. Nodes (in pink) represent DEGs and the lines (in grey) between nodes represent interactions. DEGs, differentially expressed genes. 
Table I. Top enriched biological processes for downregulated differential expressed genes in protein-protein interactions.

\begin{tabular}{|c|c|c|c|}
\hline Term & Count & P-value & FDR \\
\hline GO:0007165-signal transduction & 47 & $6.99 \times 10^{-11}$ & $1.62 \times 10^{-7}$ \\
\hline GO:0048583-regulation of response to stimulus & 37 & $6.42 \times 10^{-10}$ & $7.44 \times 10^{-7}$ \\
\hline GO:0007154-cell communication & 47 & $1.61 \times 10^{-9}$ & $7.45 \times 10^{-7}$ \\
\hline GO:0044700-single organism signaling & 47 & $1.04 \times 10^{-9}$ & $8.00 \times 10^{-7}$ \\
\hline GO:0023052-signaling & 47 & $1.43 \times 10^{-9}$ & $8.26 \times 10^{-7}$ \\
\hline GO:0050853-B cell receptor signaling pathway & 8 & $2.77 \times 10^{-9}$ & $1.07 \times 10^{-6}$ \\
\hline GO:0051716-cellular response to stimulus & 49 & $4.89 \times 10^{-9}$ & $1.62 \times 10^{-6}$ \\
\hline GO:0050896-response to stimulus & 53 & $1.31 \times 10^{-8}$ & $3.38 \times 10^{-6}$ \\
\hline GO:0002376-immune system process & 29 & $1.24 \times 10^{-8}$ & $3.61 \times 10^{-6}$ \\
\hline GO:0042113-B cell activation & 11 & $1.71 \times 10^{-8}$ & $3.97 \times 10^{-6}$ \\
\hline GO:0006955-immune response & 22 & $4.83 \times 10^{-8}$ & $9.32 \times 10^{-6}$ \\
\hline $\begin{array}{l}\text { GO:0002429-immune response-activating cell surface receptor signaling } \\
\text { pathway }\end{array}$ & 12 & $4.43 \times 10^{-8}$ & $9.32 \times 10^{-6}$ \\
\hline GO:0001775-cell activation & 17 & $1.18 \times 10^{-7}$ & $1.83 \times 10^{-5}$ \\
\hline GO:0002757-immune response-activating signal transduction & 13 & $1.27 \times 10^{-7}$ & $1.84 \times 10^{-5}$ \\
\hline GO:0050851-antigen receptor-mediated signaling pathway & 10 & $1.36 \times 10^{-7}$ & $1.85 \times 10^{-5}$ \\
\hline $\begin{array}{l}\text { GO:0002768-immune response-regulating cell surface receptor signaling } \\
\text { pathway }\end{array}$ & 12 & $1.08 \times 10^{-7}$ & $1.92 \times 10^{-5}$ \\
\hline GO:0002682-regulation of immune system process & 21 & $1.16 \times 10^{-7}$ & $1.92 \times 10^{-5}$ \\
\hline GO:0030183-B cell differentiation & 8 & $1.58 \times 10^{-7}$ & $1.93 \times 10^{-5}$ \\
\hline GO:0010646-regulation of cell communication & 30 & $1.66 \times 10^{-7}$ & $1.93 \times 10^{-5}$ \\
\hline
\end{tabular}

DEG, differentially expressed Genes; FDR, false discovery rate; GO, gene ontology.

Table II. Top enriched biological processes for upregulated differentially expressed genes in the protein-protein interaction network.

\begin{tabular}{|c|c|c|c|}
\hline Term & Count & P-value & FDR \\
\hline GO:0006928-movement of cell or subcellular component & 49 & $1.51 \times 10^{-12}$ & $2.06 \times 10^{-9}$ \\
\hline GO:0051270-regulation of cellular component movement & 33 & $1.19 \times 10^{-12}$ & $2.43 \times 10^{-9}$ \\
\hline GO:0040012-regulation of locomotion & 32 & $2.62 \times 10^{-12}$ & $2.68 \times 10^{-9}$ \\
\hline GO:0044699-single-organism process & 150 & $9.74 \times 10^{-13}$ & $3.98 \times 10^{-9}$ \\
\hline GO:2000145-regulation of cell motility & 31 & $5.04 \times 10^{-12}$ & $4.12 \times 10^{-9}$ \\
\hline GO:0048870-cell motility & 41 & $6.94 \times 10^{-12}$ & $4.73 \times 10^{-9}$ \\
\hline GO:0051674-localization of cell & 41 & $6.94 \times 10^{-12}$ & $4.73 \times 10^{-9}$ \\
\hline GO:0040011-locomotion & 43 & $3.41 \times 10^{-11}$ & $1.74 \times 10^{-8}$ \\
\hline GO:0044763-single-organism cellular process & 143 & $3.24 \times 10^{-11}$ & $1.89 \times 10^{-8}$ \\
\hline GO:0022610-biological adhesion & 45 & $6.99 \times 10^{-11}$ & $3.17 \times 10^{-8}$ \\
\hline GO:0030334-regulation of cell migration & 28 & $1.47 \times 10^{-10}$ & $6.01 \times 10^{-8}$ \\
\hline GO:0007155-cell adhesion & 44 & $2.31 \times 10^{-10}$ & $8.57 \times 10^{-8}$ \\
\hline GO:0032879-regulation of localization & 54 & $7.18 \times 10^{-10}$ & $2.44 \times 10^{-7}$ \\
\hline GO:0016477-cell migration & 35 & $1.26 \times 10^{-9}$ & $3.95 \times 10^{-7}$ \\
\hline GO:0050896-response to stimulus & 113 & $2.02 \times 10^{-9}$ & $5.89 \times 10^{-7}$ \\
\hline GO:0048518-positive regulation of biological process & 84 & $2.31 \times 10^{-9}$ & $6.30 \times 10^{-7}$ \\
\hline GO:0070887-cellular response to chemical stimulus & 55 & $2.82 \times 10^{-9}$ & $7.21 \times 10^{-7}$ \\
\hline GO:0048513-animal organ development & 59 & $2.46 \times 10^{-8}$ & $5.59 \times 10^{-6}$ \\
\hline GO:0048732-gland development & 20 & $2.37 \times 10^{-8}$ & $5.70 \times 10^{-6}$ \\
\hline
\end{tabular}

DEG, differentially expressed genes; FDR, false discovery rate; GO, gene ontology. 
Table III. Enriched Kyoto Encyclopedia of Genes and Genomes pathways for up- and downregulated DEGs in the protein-protein interaction network.

\begin{tabular}{|c|c|c|c|c|}
\hline Term & Count & Genes & P-value & FDR \\
\hline \multicolumn{5}{|l|}{ Downregulated DEG } \\
\hline $\begin{array}{l}\text { hsa04662: B cell receptor signaling } \\
\text { pathway }\end{array}$ & 10 & $\begin{array}{l}\text { CD19, FCGR2B, LYN, PLCG2, } \\
\text { CD22, CD79B, INPP5D, VAV2, } \\
\text { BLNK, SYK }\end{array}$ & $3.84 \times 10^{-10}$ & $3.84 \times 10^{-8}$ \\
\hline hsa04064: NF-кB signaling pathway & 6 & $\begin{array}{l}\text { LYN, BCL2, PLCG2, TRAF5, } \\
\text { BLNK, SYK }\end{array}$ & $2.32 \times 10^{-4}$ & $7.69 \times 10^{-3}$ \\
\hline hsa04666: Fc $\gamma \mathrm{R}$-mediated phagocytosis & 6 & $\begin{array}{l}\text { FCGR2B, LYN, PLCG2, INPP5D, } \\
\text { VAV2, SYK }\end{array}$ & $1.96 \times 10^{-4}$ & $9.77 \times 10^{-3}$ \\
\hline hsa04664: Fc $\varepsilon$ RI signaling pathway & 5 & $\begin{array}{l}\text { LYN, PLCG2, INPP5D, VAV2, } \\
\text { SYK }\end{array}$ & $9.43 \times 10^{-4}$ & $2.33 \times 10^{-2}$ \\
\hline hsa05169: Epstein-Barr virus infection & 7 & $\begin{array}{l}\text { CD19, LYN, BCL2, PLCG2, } \\
\text { ENTPD1, TRAF5, SYK }\end{array}$ & $1.33 \times 10^{-3}$ & $2.63 \times 10^{-2}$ \\
\hline hsa05200: Pathways in cancer & 9 & $\begin{array}{l}\text { COL4A3, WNT3, LPAR5, BAX, } \\
\text { BCL2, PLCG2, RASGRP2, WNT6, TRAF5 }\end{array}$ & $3.43 \times 10^{-3}$ & $4.79 \times 10^{-2}$ \\
\hline \multicolumn{5}{|l|}{ Upregulated DEG } \\
\hline hsa00480: Glutathione metabolism & 7 & $\begin{array}{l}\text { GGT6, GSTA4, RRM2, GPX3, } \\
\text { ANPEP, GSTO2, MGST1 }\end{array}$ & $1.36 \times 10^{-4}$ & $2.31 \times 10^{-2}$ \\
\hline hsa04970: Salivary secretion & 8 & $\begin{array}{l}\text { CD38, ATP1B1, CHRM3, } \\
\text { SLC12A2, AQP5, LYZ, TRPV6, PLCB1 }\end{array}$ & $3.86 \times 10^{-4}$ & $3.27 \times 10^{-2}$ \\
\hline hsa04978: Mineral absorption & 6 & $\begin{array}{l}\text { SLC11A2, TF, ATP1B1, SLC5A1, } \\
\text { TRPV6, SLC40A1 }\end{array}$ & $7.30 \times 10^{-4}$ & $4.1 \times 10^{-2}$ \\
\hline
\end{tabular}

DEG, differentially expressed gene; FDR, false discovery rate; CD, cluster of differentiation; FCGR2B, Fc fragment of IgG receptor IIb; LYN, LYN proto-oncogene Src family tyrosine kinase; PLCG2, phospholipase C $\gamma$ 2; INPP5D, inositol polyphosphate-5-phosphatase D; VAV2, vav guanine nucleotide exchange factor 2; BLNK, B-cell linker; SYK, spleen associated tyrosine kinase; BCL2, BCL2 apoptosis regulator; ENTPD1, ectonucleoside triphosphate diphosphohydrolase 1; TRAF5, TNF receptor associated factor 5; COL4A3, collagen type IV $\alpha 3$ chain; LPAR5, lysophosphatidic acid receptor 5; BAX, BCL2 associated X apoptosis regulator; RASGRP2, RAS guanyl releasing protein 2; GGT6, $\gamma$-glutamyltransferase 6; GSTA4, glutathione S-transferase $\alpha$ 4; RRM2, ribonucleotide reductase regulatory subunit M2; GPX3, glutathione peroxidase 3; ANPEP, alanyl aminopeptidase; GSTO2, glutathione S-transferase o 2; MGST1, microsomal glutathione S-transferase 1; ATP1B1, ATPase $\mathrm{Na}+\mathrm{K}+$ transporting subunit $\beta$ 1; CHRM3, cholinergic receptor muscarinic 3; SLC12A2, solute carrier family 12 member 2; AQP5, aquaporin 5; LYZ, lysozyme; TRPV6, transient receptor potential cation channel subfamily V member 6; PLCB1, phospholipase C $\beta$ 1; SLC11A2, solute carrier family 11 member 2; TF, transferrin; SLC5A1, solute carrier family 5 member 1; SLC40A1, solute carrier family 40 member 1 .

Prediction tools for the occurrence of malignancy in BLEL. In order to identify genes that could be used to predict malignant transformation in BLEL, the expression of the DEGs implicated in the pathways of cancer were compared to the expression in a control group composed of cavernous hemangioma samples. No significant difference in expression was observed for Wnt3, Wnt6, PLCG2, RASGRP2, Bcl-2, Bax, TRAF5 and COL4A3 between the control group and the BLEL group; however, the expression was significantly increased in the tissues of the malignant group $(\mathrm{P}<0.01$; Fig. 6).

\section{Discussion}

For decades, gene expression profiling was widely used to highlight the underlying transcriptional programs and molecular mechanisms between normal and malignant conditions. It was used for class discovery and prediction, or for prediction of molecular markers, and for evaluation of the response to therapeutic compounds (14-19). In the present study, microarray-based gene expression profiling was utilized to identify genes and pathways which may be associated with the occurrence of malignant lymphoma in BLEL. Comparing the expression profiles of 20,138 genes between BLEL and MLEL, 1,002 (4.98\%) DEGs were identified, among which 364 were downregulated and 638 upregulated in BLEL. In organisms, proteins interactions determine biological, molecular and cellular mechanisms which trigger the onset and progression of diseases (20); consequently, subsequent to the PPI analysis, the DEGs involved in this PPI network were used for functional enrichment of biological functions and for pathways analysis. The upregulated DEGs in the PPI network were mainly associated with the developmental processes of glands, tissues and organs, while the downregulated DEGs were associated with immune-associated signaling processes, confirming the implication of inflammation in the malignant occurrence as previously described (21). Pathway analysis identified BCR signaling and glutathione metabolism as the 


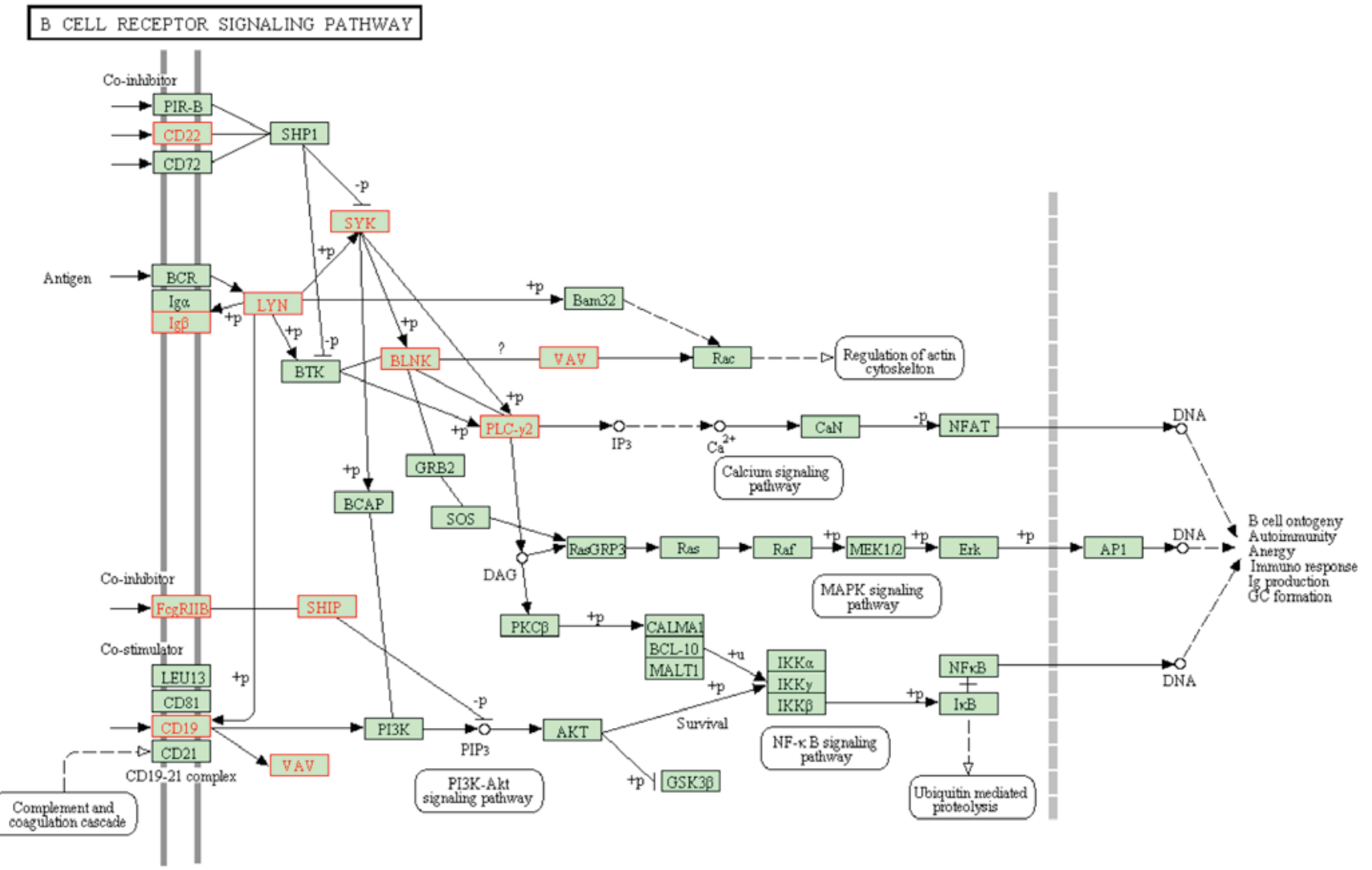

Figure 4. DEGs mapped with KEGG B cell receptor signaling pathways. DEGs involved in B cell receptor signaling pathways are labeled in red. DEGs were mapped with the KEGG and Genomes B cell receptor signaling pathways. DEGs, differentially expressed genes; KEGG, Kyoto Encyclopedia of Genes.

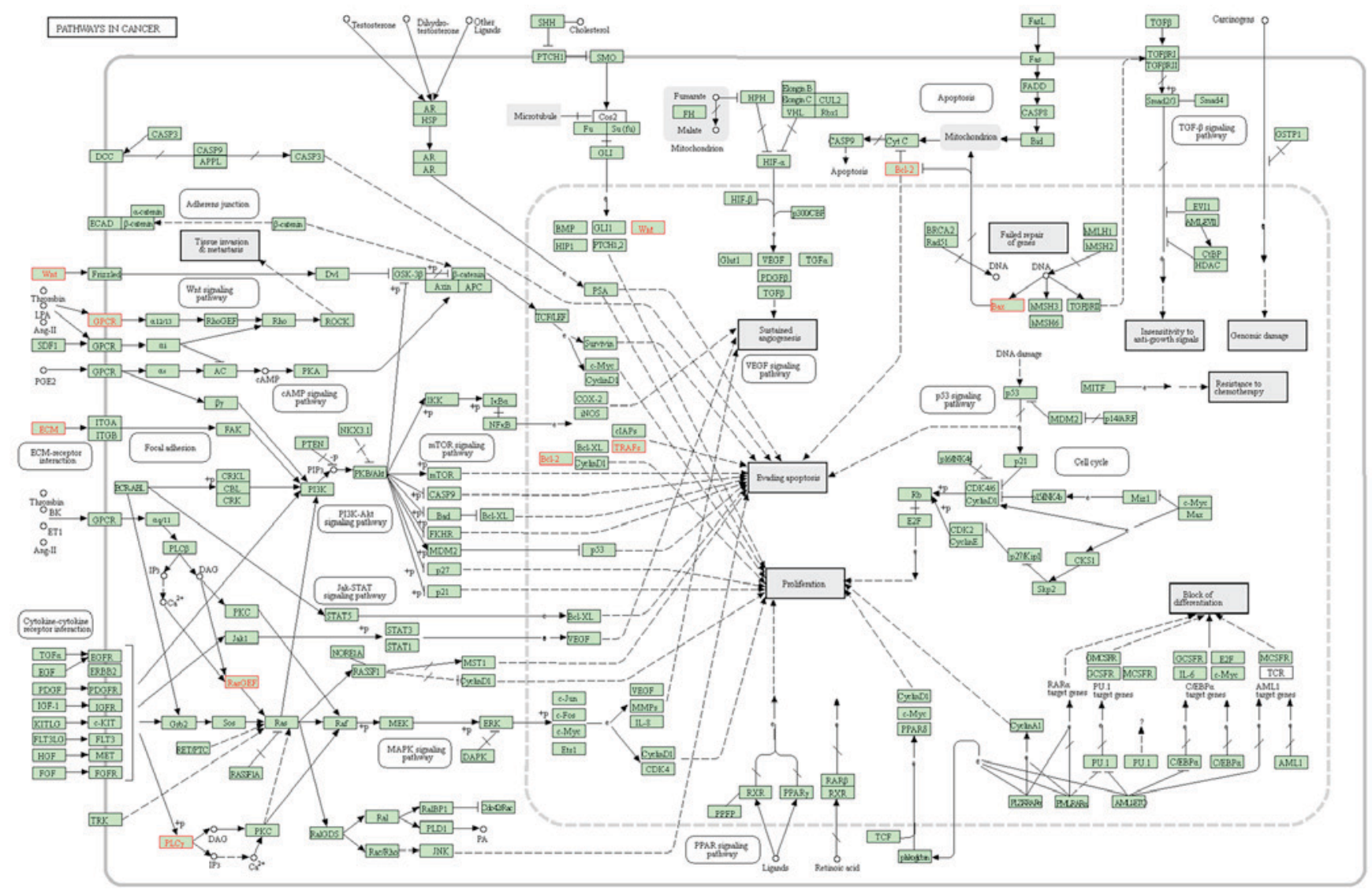

Figure 5. DEGs mapped with KEGG pathways in cancer. DEGs involved in pathways in cancer are labeled in red. DEGs were mapped with the KEGG and Genome pathways in cancer. DEGs, differentially expressed genes; KEGG, Kyoto Encyclopedia of Genes. 

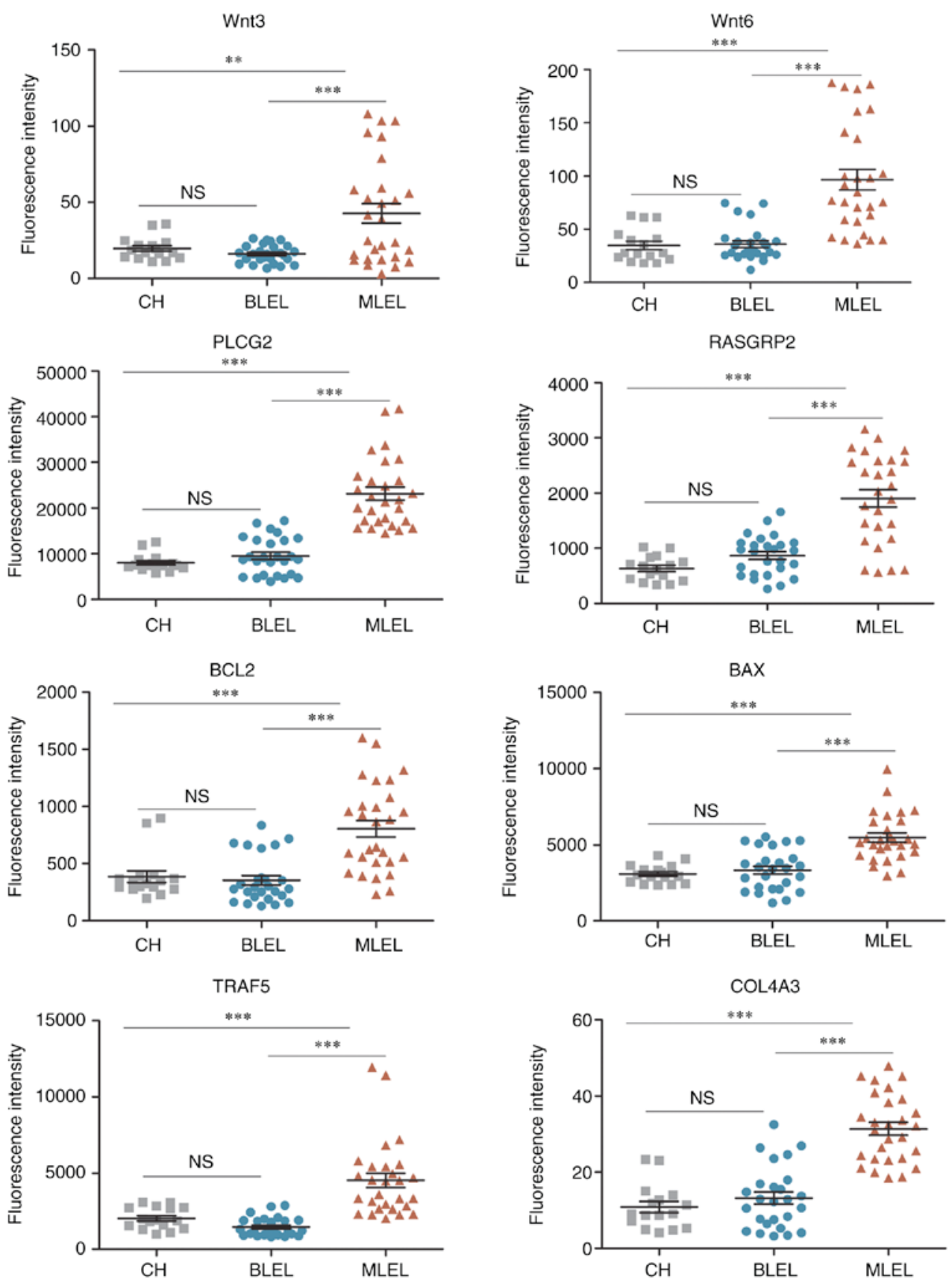

Figure 6. Expression profile of DEGs implicated in cancer pathways. All data are presented as the mean \pm standard error of the mean. ${ }^{* *} \mathrm{P}<0.01 ;{ }^{* * * *} \mathrm{P}<0.001$. $\mathrm{CH}$, cavernous hemangioma; BLEL, benign lymphoepithelial lesion; MLEL, malignant lymphoepithelial lesion; ns, not significant; DEG, differentially expressed genes; Wnt3, Wnt family member 3; PLCG2, phospholipase C $\gamma 2$; RASGRP2, RAS guanyl releasing protein 2; BCL2, apoptosis regulator BCL2; BAX, BCL2 associated $\mathrm{X}$ apoptosis regulator; TRAF5, TNF receptor associated factor 5; COL4A3, collagen type IV $\alpha 3$ chain.

most enriched pathways involving down- and upregulated DEGs, respectively, in BLEL.

Although BCR signaling is important in B-cell activation and proliferation, evidence indicates that alterations to its signalosome components may directly or indirectly contribute to lymphomagenesis $(22,23)$. Cluster of differentiation (CD) 79B overexpression was reported to induce protein kinase/mitogen-activated protein kinase 1 activation (24), decreased sensitivity to apoptosis in normal and B cell malignancies (25), and may promote lymphoma cell proliferation, while deregulation of the expression of CD19, a co-stimulator of $\mathrm{BCR}$, may accelerate malignant transformation and/or disease severity (26-28). Additionally, emerging data from clinical trials indicates that interruption of BCR signaling by tyrosine-protein kinase SYK, phosphatidylinositol 3-kinase or tyrosine-protein kinase BTK inhibitors, has substantial anti-tumor activity in a number of B-cell malignancies. Although glutathione serves important roles in a number of biological functions, including antioxidant defense, nutrient metabolism and the regulation of cellular events, deregulation of its synthesis is recognized to contribute to pathogenesis. In addition, increased glutathione levels are exhibited in a number of tumors and have been demonstrated to confer tumor resistance (29). The results of the present study indicated that these cellular pathways and associated DEGs may serve an important role in the occurrence of malignancy in patients with BLEL. 
The downregulated DEGs included a set of genes [collagen type IV $\alpha 3$ chain (COL4A3), Wnt family member 3 (Wnt-3), lysophosphatidic acid receptor, BCL2 associated $\mathrm{X}$ apoptosis regulator $(\mathrm{BAX})$, apoptosis regulator BCL2 (BCL2) phospholipase $\mathrm{C} \gamma 2$, RAS guanyl releasing protein 2 (RASGRP2), Wnt-6 and TNF receptor associated factor 5] associated with pathways in cancer. COL $4 A 3$ is located on human chromosome 2q 36-q37 (30) and has a protein distribution limited to the basement membrane with tissue specificity. COL4A3 expression is correlated with pathogenesis in gastric cancer and its high expression is correlated with a poor prognosis in lung cancer $(31,32)$. The Wnt-3/6 genes encode secreted signaling proteins, and have been implicated in oncogenesis and the regulation of cell fate. Studies of Wnt-3/6 expression suggested that they may serve an important role in carcinogenesis and their overexpression may be associated with the development of more aggressive tumors $(33,34)$. BAX and BCL2 are important regulators of cancer cell survival. The protein encoded by BCL2 is an anti-apoptotic protein; its overexpression is correlated with tumor initiation, drug resistance and a poor outcome in patients with cancer $(35,36)$. Although BAX is associated with apoptosis in a number of cell types, its heterodimerization with the anti-apoptotic protein BCL2 has been reported to suppress apoptosis (37) and to be correlated with poor clinical outcomes (38). RASGRP2 is a guanyl nucleotide exchange factor which, when malfunctioning, may contribute to blood malignancies. It increases cell viability and cell matrix adhesion via increased GTPase HRas expression and telomeric repeat-binding factor 2 -interacting protein 1 activation (39).

The comparison of the expression of the DEGs implicated in the pathways of cancer with the expression in a control group composed of cavernous hemangioma samples demonstrated no differential expression between the control group and the BLEL group; however, their expression was increased in the tissues of the malignant group, suggesting that they may be considered to be prediction tool for the occurrence of malignancy in BLEL.

In conclusion, in the present study, a set of genes and pathways were analyzed which may be useful for an improved understanding of the molecular mechanisms underlying the occurrence of malignancy in BLEL; however, further investigation is required to confirm their exact roles in this process. In addition, the potential use of certain of the identified DEGs as a predictive tool of malignant transformation is suggested.

\section{Acknowledgements}

The present study was supported by the National Natural Science Foundation of China (grant nos. 81602408 and 81371052) and the China Postdoctoral Science Foundation funded project (grant no. 143096).

\section{References}

1. Yamamoto M, Takahashi H, Sugai S and Imai K: Clinical and pathological characteristics of Mikulicz's disease (IgG4-related plasmacytic exocrinopathy). Autoimmun Rev 4: 195-200, 2005.
2. Yamamoto M, Takahashi H, Ohara M, Suzuki C, Naishiro Y, Yamamoto H, Shinomura Y and Imai K: A new conceptualization for Mikulicz's disease as an IgG4-related plasmacytic disease. Mod Rheumatol 16: 335-340, 2006.

3. Stone JH, Zen Y and Deshpande V: IgG4-related disease. N Engl J Med 366: 539-551, 2012.

4. Yamamoto M, Takahashi H, Tabeya T, Suzuki C, Naishiro Y, Ishigami K, Yajima H, Shimizu Y, Obara M, Yamamoto H, et al: Risk of malignancies in IgG4-related disease. Mod Rheumatol 22: 414-418, 2012.

5. Sato Y, Ohshima K, Ichimura K, Sato M, Yamadori I, Tanaka T, Takata K, Morito T, Kondo E and Yoshino T: Ocular adnexal IgG4-related disease has uniform clinicopathology. Pathol Int 58: 465-470, 2008

6. Go H, Kim JE, Kim YA, Chung HK, Khwarg SI, Kim CW and Jeon YK: Ocular adnexal IgG4-related disease: Comparative analysis with mucosa-associated lymphoid tissue lymphoma and other chronic inflammatory conditions. Histopathology 60: 296-312, 2012

7. Azzopardi JG and Evans DJ: Malignant lymphoma of parotid associated with Mikulicz disease (benign lymphoepithelial lesion). J Clin Pathol 24: 744-752, 1971.

8. Del Bono V, Pretolesi F, Pontali E, Martinoli C, Bassetti M, Mazzarello G, Chiaramondia M, Derchi LE and Bassetti D: Possible malignant transformation of benign lymphoepithelial parotid lesions in human immunodeficiency virus-infected patients: Report of three cases. Clin Infect Dis 30: 947-949, 2000.

9. Sato K, Kawana M, Sato Y and Takahashi S: Malignant lymphoma in the head and neck associated with benign lymphoepithelial lesion of the parotid gland. Auris Nasus Larynx 29: 209-214, 2002.

10. Ohta M, Moriyama M, Goto Y, Kawano S, Tanaka A, Maehara T, Furukawa S, Hayashida JN, Kiyoshima T, Shimizu M, et al: A case of marginal zone B cell lymphoma mimicking IgG4-related dacryoadenitis and sialoadenitis. World J Surg Oncol 13: 67, 2015.

11. Ohno K, Sato Y, Ohshima K, Takata K, Miyata-Takata T, Takeuchi M, Gion Y, Tachibana T, Orita Y, Ito T, et al: A subset of ocular adnexal marginal zone lymphomas may arise in association with IgG4-related disease. Sci Rep 5: 13539, 2015.

12. Li C and Hung Wong W: Model-based analysis of oligonucleotide arrays: Model validation, design issues and standard error application. Genome Biol 2: Research0032, 2001.

13. Lv J, Zhu B, Zhang L, Xie Q and Zhuo W: Detection and screening of small molecule agents for overcoming Sorafenib resistance of hepatocellular carcinoma: A bioinformatics study. Int J Clin Exp Med 8: 2317-2325, 2015.

14. Golub TR, Slonim DK, Tamayo P, Huard C, Gaasenbeek M, Mesirov JP, Coller H, Loh ML, Downing JR, Caligiuri MA, et al: Molecular classification of cancer: Class discovery and class prediction by gene expression monitoring. Science 286: 531-537, 1999.

15. Bullinger L, Dohner K, Bair E, Fröhling S, Schlenk RF, Tibshirani R, Döhner H and Pollack JR: Use of gene-expression profiling to identify prognostic subclasses in adult acute myeloid leukemia. N Engl J Med 350: 1605-1616, 2004.

16. Valk PJ, Verhaak RG, Beijen MA, Erpelinck CA, Barjesteh van Waalwijk van Doorn-Khosrovani S, Boer JM, Beverloo HB, Moorhouse MJ, van der Spek PJ, Löwenberg B and Delwel R: Prognostically useful gene-expression profiles in acute myeloid leukemia. N Engl J Med 350: 1617-1628, 2004.

17. Raponi M, Lancet JE, Fan H, Dossey L, Lee G, Gojo I, Feldman EJ, Gotlib J, Morris LE, Greenberg PL, et al: A 2-gene classifier for predicting response to the farnesy ltransferase inhibitor tipifarnib in acute myeloid leukemia. Blood 111: 2589-2596, 2008.

18. Kohlmann A, Bullinger L, Thiede C, Schaich M, Schnittger S, Döhner K, Dugas M, Klein HU, Döhner H, Ehninger G and Haferlach T: Gene expression profiling in AML with normal karyotype can predict mutations for molecular markers and allows novel insights into perturbed biological pathways. Leukemia 24: 1216-1220, 2010 .

19. Theilgaard-Mönch K, Boultwood J, Ferrari S, Giannopoulos K, Hernandez-Rivas JM, Kohlmann A, Morgan M, Porse B, Tagliafico E, Zwaan CM, et al: Gene expression profiling in MDS and AML: Potential and future avenues. Leukemia 25: 909-920, 2011.

20. Safari-Alighiarloo N, Taghizadeh M, Rezaei-Tavirani M, Goliaei B and Peyvandi AA: Protein-protein interaction networks (PPI) and complex diseases. Gastroenterol Hepatol Bed Bench 7: $17-31,2014$ 
21. Elinav E, Nowarski R, Thaiss CA, Hu B, Jin C and Flavell RA Inflammation-induced cancer: Crosstalk between tumours, immune cells and microorganisms. Nat Rev Cancer 13: 759-771, 2013.

22. Niemann CU and Wiestner A: B-cell receptor signaling as a driver of lymphoma development and evolution. Semin Cancer Biol 23: 410-421, 2013.

23. Refaeli Y, Young RM, Turner BC, Duda J, Field KA and Bishop JM: The B cell antigen receptor and overexpression of MYC can cooperate in the genesis of B cell lymphomas. PLoS Biol 6: e152, 2008.

24. Kim JH, Kim WS, Ryu K, Kim SJ and Park C: CD79B limits response of diffuse large $\mathrm{B}$ cell lymphoma to ibrutinib. Leuk Lymphoma 57: 1413-1422, 2016.

25. Cragg MS, Chan HT, Fox MD, Tutt A, Smith A, Oscier DG, Hamblin TJ and Glennie MJ: The alternative transcript of CD79b is overexpressed in B-CLL and inhibits signaling for apoptosis. Blood 100: 3068-3076, 2002.

26. Chung EY, Psathas JN, Yu D, Li Y, Weiss MJ and Thomas-Tikhonenko A: CD19 is a major B cell receptor-independent activator of MYC-driven B-lymphomagenesis. J Clin Invest 122: 2257-2266, 2012.

27. Li X, Ding Y, Zi M, Sun L, Zhang W, Chen S and Xu Y: CD19, from bench to bedside. Immunol lett 183: 86-95, 2017.

28. Poe JC, Minard-Colin V, Kountikov EI, Haas KM and Tedder TF: A c-Myc and surface CD19 signaling amplification loop promotes B cell lymphoma development and progression in mice. J Immunol 189: 2318-2325, 2012.

29. Lu SC: Regulation of glutathione synthesis. Mol Aspects Med 30: 42-59, 2009.

30. Morrison KE, Mariyama M, Yang-Feng TL and Reeders ST: Sequence and localization of a partial cDNA encoding the human alpha 3 chain of type IV collagen. Am J Hum Genet 49: 545-554, 1991.

31. Nie XC, Wang JP, Zhu W, Xu XY, Xing YN, Yu M, Liu YP, Takano $\mathrm{Y}$ and Zheng HC: COL4A3 expression correlates with pathogenesis, pathologic behaviors and prognosis of gastric carcinomas. Hum Pathol 44: 77-86, 2013.
32. Jiang CP, Wu BH, Chen SP, Fu MY, Yang M, Liu F and Wang BQ: High COL4A3 expression correlates with poor prognosis after cisplatin plus gemcitabine chemotherapy in non-small cell lung cancer. Tumour Biol 34: 415-420, 2013.

33. Nakashima N, Liu D, Huang CL, Ueno M, Zhang $X$ and Yokomise $\mathrm{H}$ : Wnt3 gene expression promotes tumor progression in non-small cell lung cancer. Lung cancer 76: 228-234, 2012

34. Zhang L, Yuan G, Fang Y, Qiu M, Lin J, Sun J and Yang D: Increased WNT6 expression in tumor cells predicts unfavorable survival in esophageal squamous cell carcinoma patients. Int J Clin Exp Pathol 8: 11421-11427, 2015.

35. Kaluzki I, Hrgovic I, Hailemariam-Jahn T, Doll M, Kleemann J, Valesky EM, Kippenberger S, Kaufmann R, Zoeller N and MeissnerM: Dimethylfumarateinhibits melanomacell proliferation via $\mathrm{p} 21$ and $\mathrm{p} 53$ induction and bcl-2 and cyclin $\mathrm{B} 1$ downregulation. Tumour Biol 37: 13627-13635, 2016.

36. Bajwa N, Liao C and Nikolovska-Coleska Z: Inhibitors of the anti-apoptotic Bcl-2 proteins: A patent review. Expert Opin Ther Pat 22: 37-55, 2012.

37. Yin XM, Oltvai ZN and Korsmeyer SJ: BH1 and BH2 domains of Bcl-2 are required for inhibition of apoptosis and heterodimerization with Bax. Nature 369: 321-323, 1994.

38. Mühlbeier DF, Saddi VA, de Paula ÉC, Cunha IW, Fregnani JH, Barbosa MA and Manoel WJ: Prognostic Significance of Apoptosis-related Markers in Patients With Soft-Tissue Sarcomas of Extremities. Appl Immunohistochem Mol Morphol 24: 268-274, 2016.

39. Takino J, Nagamine $\mathrm{K}$ and Hori T: Ras guanyl nucleotide releasing protein 2 affects cell viability and cell-matrix adhesion in ECV304 endothelial cells. Cell Adh Migr 7: 262-266, 2013.

This work is licensed under a Creative Commons Attribution-NonCommercial-NoDerivatives 4.0 International (CC BY-NC-ND 4.0) License. 